\title{
Coexistencia de miastenia gravis y lupus eritematoso sistémico ¿es más frecuente que lo que pensábamos?
}

\author{
Coexistence of myasthenia gravis \\ and systemic lupus erythematosus. \\ Is it more frequent than we thought?
}

$\mathrm{S}$ Editor: Motivados por un caso clínico que sistimos, revisamos las publicaciones sobre la asociación entre miastenia gravis (MG) y lupus eritematoso sistémico (LES), encontrando cambios potenciales, que nos pareció importante difundir.

Un paciente masculino de 35 años, 4 meses antes de consultar presentó disfonía, disfagia, reflujo oronasal, asociados a baja de peso de 20 kilos. Finalmente se agregaron tos y disnea progresiva, ingresando en nuestro hospital por una neumonía asociada a insuficiencia respiratoria.

En la evaluación del trastorno deglutorio por otorrinolaringólogo pesquisó paresia bilateral de cuerdas vocales. Examinado por neurólogo, objetivó ptosis bilateral fluctuante, oftalmoparesia en todas direcciones, hiporreflexia faríngea bilateral, biparesia facial, céfalo-paresia de predominio flexor y tetraparesia marcada proximal, con fatigabilidad. Se sospechó MG, lo que fue apoyado por una respuesta positiva a la prueba de prostigmina $1 \mathrm{mg} \mathrm{im}$. La electromiografía y el estudio de conducción nerviosa convencional fueron norma-

Correspondencia a: Rodrigo Aracena C. Dirección postal: Av. José Miguel Carrera 3204, San Miguel, Santiago. E mail: rodrigolili@vtr.net les. En la electromiografía de fibra única en deltoides y frontal derechos se obtuvo un Jitter aumentado, en promedio 6 veces sobre lo normal, con bloqueo inferior a $15 \%$ y densidad de fibras normal, compatible con un defecto de la transmisión neuromuscular de tipo miasténico.

Por su compromiso ventilatorio requirió manejo en unidad de cuidados intensivos.

Para la MG se usó prostigmina oral, hasta 165 $\mathrm{mg} /$ día, con pobre respuesta por lo que se indicó plasmaferesis (volumen total de recambio de 9,2 litros) y prednisona $1 \mathrm{mg} / \mathrm{kg} /$ día.

Su estudio general reveló trombocitopenia de $59.000 / \mathrm{mm}^{3}$, títulos de anticuerpos antinucleares (AAN) positivos mayor a $1 / 640$ (repetido) y proteinuria persistente no cuantificada. El estudio de anticuerpos antifosfolípidos y la tomografía computada (TC) de tórax resultaron normales.

Evaluado por reumatólogo pesquisó una historia previa de poliartralgias de pequeñas y grandes articulaciones de dos años de evolución, lo que sumado a las alteraciones del laboratorio inmunológico, hizo plantear el diagnóstico de LES.

Tras el inicio del tratamiento con prednisona se evidenció una mejoría progresiva de su condición motora, hasta la deambulación espontánea. Fue dado de alta a los 110 días en autovalencia 
parcial, con un índice de Barthel de 60, lo que se mantuvo en controles tres y seis meses posteriores al alta.

La asociación de MG a otras enfermedades autoinmunes fluctúa entre 5 y $30 \% 1,2$. Las más frecuentes son tiroiditis de Hashimoto, enfermedad de Basedow Graves y artritis reumatoide ${ }^{1,2}$.

Buscamos en la base de datos MEDLINE usando como descriptores las palabras «upus and myasthenia gravis»en el título, encontrando pocas publicaciones.

Las relaciones temporales que ocurren entre ambas entidades, se pueden agrupar en tres escenarios: presentación de ambas patologías en forma aguda al mismo tiempo, con diagnóstico simultáneo; aparición de LES en un paciente miasténico conocido y desarrollo de $\mathrm{MG}$ en un paciente con LES conocido.

La primera situación fue comunicada en dos casos $^{3,4}$ en los últimos 10 años, ambos en pacientes mujeres (12 y 36 años) que desarrollaron manifestaciones reumatológicas clínicas y de laboratorio que satisfacían los criterios del Colegio Americano de Reumatología para el diagnóstico de LES. Durante su evolución intrahospitalaria presentaron fatigabilidad muscular de extremidades y síntomas bulbares, los anticuerpos antirreceptor de acetilcolina (AchR) resultaron positivos a títulos altos y la prueba de estimulación repetitiva a baja frecuencia fue compatible con MG.

La segunda situación ha sido comunicada con mayor frecuencia, insinuándose en algunas publicaciones $^{5}$ que la timectomía actuaría como factor gatillante para el desarrollo de LES, siendo este riesgo máximo en los dos primeros años posteriores al procedimiento. La presentación clínica y evolución de estos pacientes con LES no difiere de los que no portan MG.

Dado que en nuestro paciente la clínica sugerente de LES precedió al desarrollo de debilidad generalizada, creemos que corresponde a la aparición de MG en un portador de LES (aún sin diagnóstico). En tal escenario, lo conspicuo de la debilidad, la fatigabilidad y el resultado de la electromiografía de fibra única hacen planteable el diagnóstico de MG por sobre las otras alternativas a considerar en el diagnóstico diferencial (Tabla 1).

Nuestra idea preliminar de que la coexistencia de ambas enfermedades era infrecuente, nos pareció respaldada por la comunicación reiterada de casos como la forma clínica predominante. Sin embargo, un trabajo reciente abre controversia al respecto ${ }^{6}$.

Se trata de un grupo de 78 pacientes miasténicos, en quienes se encontró $38,5 \%$ y $19,2 \%$ de títulos altos de anticuerpos antinucleares y anti ADN nativo, respectivamente. Se demostró también una incidencia de casi $8 \%$ de LES (según criterios del Colegio Americano de Reumatología) y de $6 \%$ adicional de pacientes que satisfacían 3 criterios para el diagnóstico de LES; dichos pacientes habían recibido diversos tratamientos inmunosupresores para su MG, planteando los autores que esto posiblemente enmascaró la expresión clínica del LES.

Esta información cuestiona que la asociación de ambas enfermedades sea excepcional y plantea la necesidad de investigar un LES en pacientes miasténicos, en especial si se trata de mujeres con inicio precoz de la enfermedad ya que constituían la mayoría de los casos del estudio señalado.

La importancia clínica de establecer tempranamente la asociación, radica en el potencial enmascaramiento mutuo que podría generar la inmunosupresión empleada en el tratamiento de ambas entidades. En la situación de LES en miasténicos, posiblemente requerirán mayores niveles de inmunosupresión para evitar el daño sistémico del LES. De igual modo, la MG debería incluirse sistemáticamente en el diagnóstico diferencial de un paciente con LES que desarrolla debilidad y fatigabilidad muscular por las impli-

\section{Tabla 1. D iagnóstico diferencial en un paciente portador de LES y debilidad generalizada}

Avance del propio Lupus
Polimiositis
Dermatomiositis
Miopatía distiroidea
Miopatía por fármacos:
$\quad$ * Esteroidal
$\quad$ *Cloroquina
Miastenia gravis
Síndrome de Eaton-Lambert


cancias que significa esta condición, en cuanto a conducta de estudio y manejo (anticolinesterásicos, timectomía e inmunosupresión), así como de

\section{REFERENCIAS}

1. Christensen PB, Jensen TS, Tsiropoulos I, Sorensen T, KJaER M, Hojer-Pedersen E et al. Associated autoimmune diseases in myasthenia gravis. A populationbased study. Acta Neurol Scand 1995; 9: 192-5.

2. Rastenyte D, Vaitkus A, Neverauskas R, Pauza V. Demographic and clinical characteristics of patients with myasthenia gravis. Medicina (Kaunas) 2002; 38: 611-6.

3. Nishimura A, Yamazaki $\mathrm{H}$, Fuchigami T, Fujita $\mathrm{Y}$, O kubo O, HaRada K et al. [A childhood case of systemic lupus erythematosus associated with myastheniagravis] No To Hattatsu 1997; 29: 390-5. educación al paciente sobre los factores agravantes de su condición (fármacos, cirugía, infecciones) presentes en la vida cotidiana.

\section{Rodrigo Aracena C, Jorge Nogales-Gaete}

Servicio de Neurología, Complejo Asistencial Barros Luco y Departamento de Neurología Sur, Facultad de Medicina de la Universidad de Chile. Santiago de Chile.

4. Barbosa RE, Córdova S, Cajigas JC. Coexistence of systemic lupus erythematosus and myasthenia gravis. Lupus 2000; 9: 156-7.

5. Bhinder S, Majithia V, Harisdangkul V. Myasthenia gravis and systemic lupus erythematosus: truly associated or coincidental-two case reports and review of the literature. Clin Rheumatol 2005; 18: $1-2$.

6. Sthoeger Z, Neiman A, Eubirt D, Zinger H, Magen E, BURSTEIN R ET AL. High prevalence of systemic lupus erythematosus in 78 myasthenia gravis patients: a clinical and serologic study. Am J Med Sci 2006; 331: 4-9. 